\title{
Investigation of the Ocean Thermal Energy Conversion and Published Energy Source: Theory and Experiment
}

\author{
Zhihao Li, Jiapeng Su, Anjun Jin* \\ The Maritime Faculty, Ningbo University, Ningbo, China \\ Email address: \\ ajjin@nbu.edu.cn (Anjun Jin) \\ ${ }^{*}$ Corresponding author
}

To cite this article:

Zhihao Li, Jiapeng Su, Anjun Jin. Investigation of the Ocean Thermal Energy Conversion and Published Energy Source: Theory and Experiment. American Journal of Science, Engineering and Technology. Special Issue: Advances in Thermoelectric Generation and Renewable Energies. Vol. 5, No. 2, 2020, pp. 105-109. doi: 10.11648/j.ajset.20200502.18

Received: MM DD, 2020; Accepted: MM DD, 2020; Published: June 18, 2020

\begin{abstract}
There is a great need for Clean Energy Technology. The ocean is a vast resource for improvement of the energy supply. The ocean thermal energy conversion (OTEC) is clean and renewable, the power generation is stable, and the energy storage has high capacity. Furthermore, authors construct a mathematical model to study the maximal available benefit for a prosumer. The prosumer, for the most part, expends energy of its own and utilizes an incorporated energy framework that occasionally produces energy for other customers. The ocean thermal energy conversion (OTEC) framework is one of the steady energy sources in published energy source (PES), also known as energy blockchain (EBC). Firstly, the restriction on a traditional OTEC is ordinarily at about $1 \%$ that can be overcome with an improved efficiency at $10 \%$ or higher. Second, the contextual investigation should shed light on the demonstration and system produced for PES or EBC. Third, a precise investigation uses the EBC input parameters as follows: OTEC-based multi-energy complementarity, energy storage/ power parameter, and the smart grid power. This model connects the electric load to the external grid power through a gate-control that has a smart meter inside the prosumer's EBC framework. All these parameters may be time variable. Fourth, the EBC output can incorporate the following variables: 1) Energy storage, 2) Financial target output, and 3) P2P energy transaction as indicated by the PES eco-framework. Finally, this study is to present PES properties in the view of the value dependency law. The output and its optimization are to resolve the dependency law upon input parameters referenced at above. The optimum solution increases the benefit for the prosumer.
\end{abstract}

Keywords: Energy Blockchain, Published Energy Source, Peer to Peer, Renewable Energy, Ocean Thermal Energy Conversion, Energy Storage, Solar Complementary Heat, Open-Cycle, Closed-Cycle OTEC

\section{Introduction}

The modern world is weary of one-off traditional energy resource and of the aggravated causes of climate change; there are complex deficiencies or polymorphic circumstances in order to address the related root causes [1]. The ascent of renewable energies and the rapid advancement of communication innovation are great opportunities to address the aforementioned causes. There is interesting progress to resolve the above challenges facing the future of mankind [2, 3]. The utilization of technology advancement to resolve prior deficiencies in traditional energy, to mitigate climate abnormalities and to reduce potential pandemic environment has been the quest of many scientists. Many exciting technological advancements in the published energy source can be very valuable due to that the technology can readily benefit the users with significantly innovative solutions.

First off, because of the dual necessities of climate change and ecological protection, the traditional energy structure ruled by fossil-based energy, for example, coal, oil, and gas is steadily being questioned due to their poor consequential carbon emission [4]. This traditional structure of energy is recently expanded and/or replaced to include the alternative energies; for example, the solar photovoltaic and wind powers have been playing important roles to date. The alternative energy structure depends on a alternative power framework [5]. For example, the solar emerging technology has recently achieved very good development with significantly 
improvement of efficiency, great cost, and potential stability [6]. The alternative power framework has characteristic points of demand that are has more under digitalization than its counterparts with fossil energy in nature [6]. Moreover, the recent rapid development and emphasis of artificial intelligence, big information, cloud computing, the Internet of things and various other advances, delivers a computing infrastructure for the digitalization of traditional industries that gives substantial boost for the ascent of digitalization in the energy industry.

\section{Development of the Ocean Thermal Energy Conversion System}

According to the statistics, the temperature of the deep seawater $1000 \mathrm{~m}$ below 24 -degree latitude from the equator is about $4^{\circ} \mathrm{C}$, and the temperature of the sea surface water is about $30^{\circ} \mathrm{C}$. Song et al [7] shows that the available energy from the thermal energy between the deep-sea and sea surface waters is about $1.013 \mathrm{TW}$. The ocean may potentially generate a significant amount of energy. The annual energy is projected to add up to 87600TWh [8]. In comparison, the global annual electricity demand is about $16000 \mathrm{TWh}$. The ocean thermal energy is renewable according to the law of Physics on both the earth gravity and the surface water heating due to the normal solar irradiation $[2,8]$. In addition to be renewable, the ocean thermal energy conversion (OTEC) provides stable, clean and pollution-free energy; it is extremely valuable for further development. Due to that the clean energy is a driving factor, recent exploration is dedicated to significantly improve the energy conversion efficiency and to lower the related cost. Figure 1 illustrates a design of the typical OTEC system.

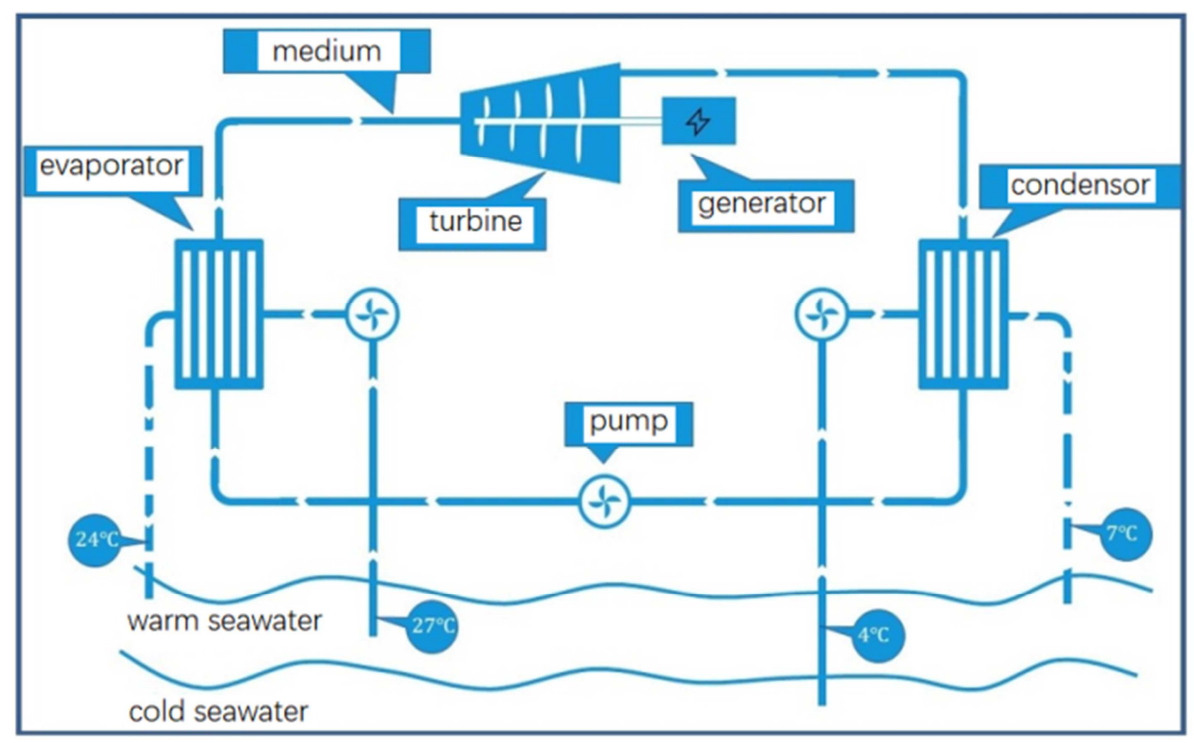

Figure 1. Schematic illustrates the ocean thermal energy conversion.

The working media fluid could be the warm seawater in open-cycle operation and/or the ammonia-based media in closed-cycle operation that has low-boiling point. The cycles drive a Carnot heat engine thermodynamically between warm seawater and cold deep seawater. A couple of examples of the world's top OTEC demonstration facilities are the Makai system in USA and the Okinawa system in Japan [9]. The world's first production of net electrical power via ocean thermal energy conversion took place at the Natural Energy Laboratory of Hawaii Authority (NELHA) in Kona on Hawaii Island following the 1970s national energy crisis. Currently the Makai OTEC system continues to drive practical ways in employing various ocean energy [9].

Makai's renewable energy lab has explored many practical ways of harnessing the abundance of natural energy present in the ocean. Makai has employed the existing and/or developing technologies such as: power generation: ocean thermal energy conversion; wave, current, OTEC. The OTEC power is at multi mega-watt (MW) level and has operated for over four decades since the launch.
Traditionally the OTEC is limited to the small-scale demonstration due to the reasons as follows. 1) The efficiency of traditional single ocean thermal energy conversion system is low, which is generally $1 \%[10] ; 2$ ) it is challenging and expensive to extract cold seawater from the $800 \mathrm{~m} \sim 1000 \mathrm{~m}$ deep sea; 3 ) at the same time the deep ocean water is released into the surface ocean, microbes from the bottom are released into the ocean surface that may trigger changes in the ecosystem. Nevertheless, the research progress shows that the efficiency can overcome the above limit and that the conversion efficiency can achieve at $10 \%$ for solar heated open-cycle OTEC process within a small margin of error. The warm seawater can be stored easily. Researchers have employed the closed cycle operation with working media such as ammonia that has low boiling point to move the turbines. The surface seawater can increase its temperature with additional solar heating. With the improved efficiency, net energy generation, and the eco-system solution, the OTEC is very attractive that can be an advantageous solution for the distributed power source. 
The renewable energy source system, such as solar photovoltaic and wind power, is vulnerable to environmental instability. The electricity generation is unstable, which hinders its further development. The multi-energy system based on OTEC, along with the above renewable energies, can stabilize the generation. Moreover, the stability needs usually require the balance in local consumption, energy storage, and/ or grid connection. The application and development of energy storage technology will solve the problems of access and consumption of distributed generation sets. Energy storage device is equipped which renders much better stability by cutting peak and filling valley of electric usage, energy scheduling and smoothing fluctuations.
Shown in Figure 2 is an illustration of the multi-energy generation based on OTEC. Referring to Figure 2, the energy storage has achieved multi mega-watt hours by means of serially and parallelly connected batteries. The lithium batteries have been around since the invention by a Japanese researcher Yoshino. The lithium batteries are employed in various industries as follow: the automotive industry which achieves safe driving, energy storage system, the IoT devices, and the infrastructure industry which support daily life. To meet the operational safety requirements, it is usually equipped with smoke fire sensor, video monitoring system, fire protection system and air conditioning system.

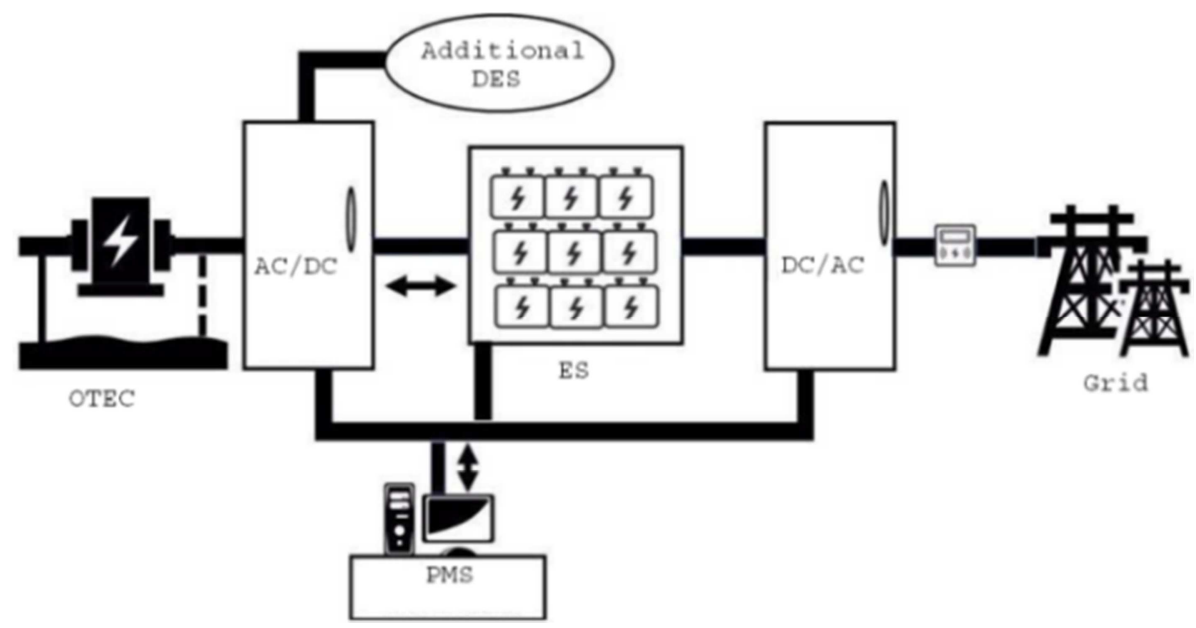

Figure 2. Shown is a schematics of a distributed energy system equipped with multi-energy generation, OTEC, and energy storage.

There are various energy storage solutions available. For example, Wang., et al. [11], provide a hybrid hydrogen energy technology to split the water chemically and store the energy. The Wang's team at Pacific Northwest National Laboratory has a number of inventive works who claims the hybrid hydrogen energy to perform better in the lab by utilizing a type of fuel cell acting both as battery and hydrogen generator than the current commercial products.

\section{Mathematical Studies of the Published Energy Source Model}

Blockchain innovation has started from an establishing paper "Bitcoin: a peer-to-peer electronic money framework". The paper was published in 2008 by scientists under the pseudonym Satoshi Nakamoto [12]. In 2009, the bitcoin transaction framework was formally conceived, and blockchain innovation progressively entered the public eye. With the surge of demand for bitcoin in 2016 and the quick development of demand for bitcoin, the investigation on the original blockchain innovation has quickly expanded. Its applications have stretched out from the original digital money to finance, the Internet of things, smart manufacturing, etc. $[13,14]$.

A white paper by the Ministry of IIT, China, includes the key innovations context of the blockchain technology. Based on the literature [15], blockchain innovation is another decentralized infrastructure and published processing paradigm that utilizes cryptographic blockchain structure to check and store information, that utilizes published node contract algorithm to generate and update information, and that utilizes robotized script code (smart contract) to program and control information.

The PES approach is tremendously useful that governs a prosumer's value dependency law and that is worked out with an application at below. Specific input parameters include the following: the rate of power generation (PG) such as OTEC and multi-energy complementarity, local consumption of Power (CP); and the feed-in of grid power (GP). Moreover, the energy storage is included in the application. Among several output variables, the one solution to solve for is the financial saving and earning (SE), the benefits of energy ES, and peer-to-peer transaction.

The input parameters are measured data and has time variance. These parameters are monitored daily and have weighted average to influence the output. The output variables, e.g., Fin, deliver results to a prosumer on $\mathrm{CP}$ who can make decisions based on the following equation and the data from the input parameters.

$$
F i n(t)=\int d t\left\{\left[P G * \alpha_{1}(t)-C P * \alpha_{2}(t)\right] \times p(t)+E S \times \alpha_{3}(t) \times \Delta p-G P * \alpha_{4}(t) \times p(t)\right\}
$$


Various input parameters are the measured data that have time dependency, and that is integrated over the time as shown in eq. (1).

The PG is an input parameter, e.g., of the total solar PV generation (from specification); with ${ }_{1}(\mathrm{t})$ being an ambient power coefficient that has a range of $(0,1)$ and varies with time during the day. $\mathrm{p}(\mathrm{t})$ is a scheduled price from utility provider [16].

$\mathrm{CP}$ is an input parameter dictated by a prosumer who decide how many in total household power to use for what time in which day. In eq. (1), CP is multiplied by ${ }_{2}(t)$ where

${ }_{2}(\mathrm{t})$ is a parameter that ranges $(0,1)$ for the distributed power deployment or that may be a customer's specification.

Normally the ES charges energy at the night and discharges in the day. The energy level of ES ranges by the manufacturer's spec, e.g., between $10 \%$ to $95 \%$ during a discharge-charge cycle.

The coefficient ${ }_{3}(\mathrm{t})$ in ES is the effective chargedischarge rated coefficient that ranges $(-1,1)$. Its actual range may depend on the manufacturer's specification. $p$ is the power bill for peak and valley differential in scheduled price.

GP represents the grid power input that is a parameter for prosumer to decide. In eq. (1), the term GP is multiplied by

${ }_{4}(\mathrm{t})$; where ${ }_{4}(\mathrm{t})$ is an ambient power coefficient that ranges $(0,1)$. A smart meter connects EBC to the external grid. $p(t)$ is the scheduled price of electricity.

There are separate studies; Lu et al [16] have previously found that each of the photovoltaic capacity and the energy storage capacity in deliverable power should be higher than the peak power of the family use.

A typical hardware structure delivers the important input parameters of the system. Inside a local access network (LAN) in particular, the Energy LAN framework, the power supply side can be made of wind turbine, solar photovoltaic (PV) unit, OTEC, energy storage framework and the external power matrix. The power demand side is made of the traditional load, power transaction, and published available energy storage. Under the activity of the automated demand reaction mode, the demand-side energy storage coordinates its various variables and operation algorithm. Under the condition that the inventory of renewable energy is equal to or higher than the traditional load demand, the energy storage framework may decide and react to the framework by charging or discharge the ES.

As indicated by its charge condition, the energy storage framework can alter its charging and discharging mode to compensate the change in renewable energy output, to load demand and electricity transaction cost, to improve the use effectiveness of renewable energy and load levelling of the grid. Under the condition of obtaining power from the power grid; the published capacity of PES is dependent upon the present energy storage condition. Power control system (PCS, or power management unit) is a bidirectional energy transference mechanism that causes energy transaction between the energy storage framework and the power grid. It is equipped with all universal communication interface, who acknowledges charge and discharge mode switching, and power control function by accepting the control strategy issued by an upstream controller. What's more, it can execute digital command by a battery management system in the downstream. Smart electric meters acknowledge two-way metering, recording both energy storage consumption and power output.

The energy storage is designed with its end goal that provides stable power. On account of multi-energy generation, the accessible power adjustment at peak and valley could benefit the prosumer and earn the price differential; the energy storage unit is significant for both energy use and levelling load. Authors have indicated that the OTEC-based PES and EBC innovation can accomplish an objective that meets smart and stable power output, transparent, secure, and self-repairment features.

Let us work out a design recommendation at below. Authors have investigated a multi-energy complementary system of solar photovoltaic, OTEC, prosumer's consumable power, energy storage, and grid power. The output level is at a mega-watt level. Governed by the value dependency law, a typical solution is shown at Table 1 at below.

Table 1. Simulation results show a design analysis of the PES with a multi-energy complementary system.

\begin{tabular}{|c|c|c|c|c|}
\hline PV $(K W)$ & OTEC $(\mathrm{KW})$ & $\mathrm{CP}(\mathrm{KW} / \mathrm{KW})$ & ES (MWh/MW) & GP (KW) \\
\hline 800 & 1600 & $2400 / 300$ & $5.0 / 1$ & 500 \\
\hline 1200 & 1600 & $2400 / 300$ & $5.0 / 1$ & 1000 \\
\hline 800 & 2000 & $2400 / 300$ & $5.0 / 1$ & 1500 \\
\hline 1600 & 2000 & $2400 / 300$ & $5.0 / 1$ & 2000 \\
\hline
\end{tabular}

Where PV, OTEC, and GP data units are in $\mathrm{KW}$; the unit of the consumer power load peak/valley is in MW. The unit of ES configuration for either peak or valley in the table is MWh. Simulation yields typical monthly savings and earnings in hundreds of dollars, USD. The designs of the multi-energy sources can be readily scaled up.

\section{Conclusions}

Within a digitization framework, a power management unit issues command information that manages energy intake and output for an energy block chain (EBC). The prosumer can operate his/her EBC to deliver ROI with optimal (maximum) benefit. The EBC digitization is technologically advantageous for the value adding and the peer-to-peer transaction with alternative energies. The multi-energy complementarity is employed to balance the energy and its overall availability. The ocean thermal energy conversion (OTEC) provides the base electricity and stabilizes the base power; OTEC has net power output and has achieved significantly improved 
efficiency. OTEC can be suitable for the commercial competitiveness. This article is to offer valuable insights for design and development of the PES with OTEC-based multi-energy system. The results show that the complementary system of OTEC can improve the optimal power generation, conversion efficiency, stability, output level, and ocean energy usage.

Finally, along with the energy storage, authors report detailed studies of published energy source (PES) and provide the prosumer a value dependency law that can derive maximal value and maximum efficiency. They discover that the PES law for maximal ROI can deliver best the value and stable power.

\section{Acknowledgements}

Authors are appreciative of discussions with Prof. Y. Han, Jing Wang, Zhao Ma, Hui Yu. Their discussions are helpful for studies in this article.

\section{References}

[1] The Paris Agreement. https://unfccc.int/process-and-meetings/ the-paris-agreement/the-paris-agreement. [accessed 10 Jun 2019]. Google Scholar.

[2] The Illustration, LXXXVII (13 July 1929) 34-35. The New York Times, June 6, 1632.

[3] Karottki G D, Spilak M P, Frederiksen M, et al (2015). Indoor and Outdoor Exposure to Ultrafine, Fine and Microbiologically Derived Particulate Matter Related to Cardiovascular and Respiratory Effects in a Panel of Elderly Urban Citizens. International journal of environmental research and public health, 12 (2): 1667-1686. Google Scholar.

[4] Ford G, Niblett C, Walker L (1983). Ocean thermal-energy conversion. Science, Measurement and Technology, IEEE Proceedings A, 130 (2): 93.

[5] Energy Union Package. A framework strategy for a resilient energy union with a forward-looking climate change policy.
https://eur-lex.europa.eu/legal-content/en/TXT/?Uri=COM\%3 A2015\%3A80\%3AFIN [access 9 Nov 2019] Google Scholar.

[6] Zhihao Li, Kuan W. A. Chee, Zhenhai Yang, Jiapeng Su, Jiapei Zhao, Anjun J. Jin (2020), Review of an Emerging Solar Energy System: The Perovskite Solar Cells and Energy Storages; Adv. Mater. Lett, 11 (5), 20051505.

[7] Song Y (2019). A study of OTEC application on deep-sea FPSOs. Journal of Marine Science and Technology, 24 (2): 466-478. Google Scholar.

[8] Khan N, Kalair A, Abas N, et al (2017). Review of ocean tidal, wave and thermal energy technologies. Renewable \& Sustainable Energy Reviews, 72: 590-604.

[9] Makai Ocean Engineering working with Navy on Big Island OTEC project. https://www.bizjournals.com/pacific/news/2013/03/19/makaiocean-engineering-working-with.html [accessed 10 Jun 2019].

[10] Heydt, G. T (1993). An assessment of ocean thermal energy conversion as an advanced electric generation methodology. Proceedings of the IEEE, 81 (3): 409-418.

[11] Wang W. U.S. Department of Energy's ARPA-Energy, https://noise.getoto.net/2020/04/13/redox-flow-cell-stores-ren ewable-energy-as-hydrogen/2/.[accessed 1 Apr 2020].

[12] Nakamoto S. Bitcoin: A peer-to-peer electronic cash system. https://bitcoin.org/bitcoin.Pdf. [access 30 Dec 2019] Google Scholar.

[13] Eyal, Ittay (2017). Blockchain Technology: Transforming Libertarian Cryptocurrency Dreams to Finance and Banking Realities. Computer, 50 (9): 38-49.

[14] Huckle S, Bhattacharya R, White M, et al (2016). Internet of Things, Blockchain and Shared Economy Applications. Procedia Computer Science, 98: 461-466.

[15] Yong Y, Feiyue W (2016). Blockchain: the state of the art and future trends. Acta Automatica Sinica. 42 (4): 481494. (China)

[16] LU Qing, ZHANG Zhixin, LENG Yajun, LYU Shuaikang (2019). Scheduling Model of Smart Home Electricity Consumption System Considering Photovoltaic Energy Storage. New Energy, 47 (12): 30-36. China. 